\title{
Cintigrafía de Vía Biliar en el Síndrome Ictérico del Lactante Menor
}

\author{
Dr. Fernando Betancourt M.1, Dr. Francisco Larraín B. ${ }^{2}$. Dr. Patricio González E. ${ }^{\text {, }}$ \\ Dr. Enrique Olea G. ${ }^{3}$, Dr. Enrique Lillo G. ${ }^{3}$, Dr. Gustavo Aldunate N. ${ }^{4}$, Dr. Osvaldo Danús V. ${ }^{2}$
}

\section{Hepatobiliary Scintigraphy with Tecnetium $99 \mathrm{~m}$ in Infantile Obstructive Cholangiopathy}

\begin{abstract}
Hepatobiliary semtigraphy with labcled tecnetium $99^{\text {tn }}$ was used to evaluate 7 infants with mixed jaundice in order to rule out biliary atresia. The patients ranged in age from 7 days to 4 months.

In 6 patients there was no evidence of excretion of the tracer into the intestinal tract. In all 6 , there were clinical manifestations of complete biliary obstruction. One patient with clinical picture of partial bitiary obstruction, showed definite excretion.

The authors conclude that hepatobiliary scintigraphy with labeled Tecretium $99 \mathrm{~m}$, is a good test to study the permeability of the biliary tract although it does not yield better information than that obtained with the nomal clinical methods.
\end{abstract}

El síndrome ictérico en el lactante menor constituye siempre un gran desafío para el pediatra tanto por la multiplicidad de etiologias que entran en juego, como por la dificultad diagnóstica entre estos diferentes factores. Además continúan siendo problemas de la más alta importancia la terapéutica oportuna y racional que debiera ser aplicada asi como la alta letalidad que presenta esta patología 1-2-3-4.

Estos aspectos adquieren su máxima expresión cuando se estudia el síndrome ictérico de tipo obstructivo, en los primeros meses de la vida. Aunque en el último tiempo ha sido posible disponer en forma progresiva de técnicas avanzadas tendientes tanto a diferenciat las diversas etiologias, así como el grado de permeabilidad del árbol biliar, el problema no parece haberse aclarado en absoluto y persisten múltiples interrogantes en relación a las dificultades planteadas ${ }^{5-6-7-8}$

En esta oportunidad se presenta el estudio y los resultados obtenidos en 7 lactantes menores de 4 meses con sindrome ictérico de tipo obstructivo. Estos pacientes ingresaron en forma consecutiva en periodo de 6 meses a la Unidad de Gastroenterología del Hospital Roberto del Río y en ellos se utilizó, por primera vez en nuestro medio en este tipo de patología, el estudio cintigráfico de via biliar.

1 Médico. Becado Unidad de Gastroenterología. Hosp. R. del Río.

2 Médico. Unidad de Gastroenterología.

3 Médico. Unidad Medicina Nuclear. Hosp. J.J. Aguir re.

4 Médico. Servicio de Cirujía. Hosp. R. del Río.

\section{MATERIAL Y METODO}

El grupo está constituido por 7 lactantes cuyas edades al ingreso variaban entre 7 dias y 4 meses. Tres hombres y cuatro mujeres. En cinco, el sindrome ictérico presentaba características clínicas de obstrucción total y en dos era parcial.

En todos ellos además de la recopilación de antecedentes de anamnesis y examen físico, se realizó estudio tendiente a descartar Lues, Toxoplasmosis, Chagas, Inclusión Citomegálica, Infección urinaria. Se hizo determinación de bilirrubinemias seriadas cada 6 días, transaminasas, estudio de coagulación, presencia de HBsAg, y estudio histológico hepático por medio de punción biopsia. Tanto los antecedentes como los resultados obtenidos se presentan en la Tabla 1.

En los 7 pacientes y en un lactante sano de 3 meses de edad, se hizo estudio cintigráfico de la via biliar con Tecnecio $99 \mathrm{~m}$ DISIDA. Antes del examen fueron tratados con fenobarbital oral a dosis de $5 \mathrm{mg} / \mathrm{Kg} /$ dia durante 7 días. Con los pacientes en ayunas se inyectó por vía endovenosa 50 Microcuries por $\mathrm{Kg}$ de peso de Tecnecio $99 \mathrm{~m}$ con una dosis mínima de 1 Milicurie. Se registraron proyecciones anteroposterior, posteroanterior y laterales en gamacámara OHIO 410 Sigmá cada 15 minutos durante las primeras dos horas. Controles entre las 4 y 6 horas, a las 24 horas y 48 horas.

En los pacientes en que el estudio cintigráfico reveló obstrucción de la vía biliar (6 casos) se practicó colangiografía transparieto-hepática y exploración quirúrgica de la vía biliar extrahepática. 
Tabla 1.

Antecedentes en 7 lactantes con sindrome de ictericia obstructiva

\begin{tabular}{|c|c|c|c|c|c|c|c|}
\hline $\begin{array}{c}\text { Caso } \\
\text { No/Sexo }\end{array}$ & Antecedentes & $\begin{array}{l}\text { Inicio Sínd. Obstr. } \\
\text { (Días) }\end{array}$ & $\begin{array}{c}\text { Edad al Ingreso } \\
\text { (Días) }\end{array}$ & $\begin{array}{l}\text { Bilirrubina } \\
\text { Total MG' } \\
\text { D.A 1 \% }\end{array}$ & $\begin{array}{c}\text { Transaminasas } \\
0 / \mathrm{P}\end{array}$ & $\begin{array}{l}\text { Reacciones } \\
\text { Torch }\end{array}$ & $\begin{array}{l}\text { Respuestas } \\
\text { F.B. }\end{array}$ \\
\hline $1 / \mathrm{M}$ & $(-)$ & 30 & 75 & $15 / 48$ & $210 ; 160$ & $(-)$ & $\mathbf{F}$ \\
\hline $2 / \mathrm{F}$ & $(-)$ & $?$ & 45 & $19 / 51$ & $380 / 160$ & $(-)$ & $(-)$ \\
\hline $3 / \mathrm{F}$ & $(-)$ & 30 & 35 & $9 / 56$ & $210 ; 120$ & $(-)$ & $(-)$ \\
\hline $4 / F$ & $\begin{array}{l}\text { Pretérmino } \\
\text { Lues Mat. } \\
\text { sepsis N.N. }\end{array}$ & 7 & 120 & $8 / 51$ & $119 / 90$ & $(-)$ & $(-)$ \\
\hline $5 / F$ & $(-)$ & 30 & 45 & $20 / 50$ & $190 / 142$ & $(-)$ & $(-)$ \\
\hline $6 / \mathrm{M}$ & $\begin{array}{l}\text { Sindrome Down } \\
\text { Enf. Hemolitica }\end{array}$ & $\begin{array}{l}\text { Parcial } 5 \\
\text { Total } 24\end{array}$ & 7 & $7 / 38$ & $140 / 80$ & $(-3$ & $(-)$ \\
\hline $7 / \mathrm{M}$ & $\begin{array}{l}\text { Síndrome Down } \\
\text { Sepsis N.N. }\end{array}$ & Parcial 7 & 21 & $6 / 48$ & 75170 & $(-)$ & $(-)$ \\
\hline $8 / F$ & Control & - & - & - & . & - & - \\
\hline
\end{tabular}

\section{RESULTADOS}

Los resultados obtenidos en el estudio histológico hepático, cintigrafía de la vía biliar, colangiografía transparieto-hepática y exploración quirürgica se presentan en la Tabla 2.

El estudio histológico del hígado reveló alteraciones compatibles con Hepatitis Neonatal en 3 pacientes: en 2 las-lesiones eran acentuadas con características de Hepatitis Crónica (Casos 1 y 5) y 1 leve (Caso 7). En otros 2 lactantes se observó sólo alteraciones inflamatorias localizadas en los espacios porta (Caso 2 y 3 ) presentando uno de ellos evidencias de cirrosis biliar en desarrollo. En el lactante de mayor edad (Caso 4) existía una cirrosis biliar franca y en el Caso 6 , portador de

Tabla 2.

Resultados en 7 lactantes con ictericia obstructiva

\begin{tabular}{|c|c|c|c|c|c|c|}
\hline Caso & Sirid. Obsiruct. & $\begin{array}{c}\text { Tiempo Evol. } \\
\text { (Mes) }\end{array}$ & Biopsis hepitica & Cintigrafja & Colangiografía & Laparotomia \\
\hline l & Tocal & 2 & Hepatitis crónica & $(-)$ & $(-)$ & $\begin{array}{l}\text { Higzdo cirrotice } \\
\text { Vesicula atrolica } \\
\text { Colćdoco: ( ) } \\
\text { Adenopatías }\end{array}$ \\
\hline 2 & Total & b & $\begin{array}{l}\text { Infiltración } y \\
\text { proliferación } \\
\text { espacio ports }\end{array}$ & $i-)$ & $(-)$ & $\begin{array}{l}\text { Vesícula atrófica } \\
\text { Coledoco: Cordón } \\
\text { Fibroso } \\
\text { Hepáticos: Atróficos }\end{array}$ \\
\hline 3 & Toral & 1 & $\begin{array}{l}\text { Intiltración y } \\
\text { proliferación } \\
\text { espacio porta, } \\
\text { cirrosis biliar }\end{array}$ & $(\cdot-)$ & $(-)$ & $\begin{array}{l}\text { Vía biliar } \\
\text { Externa } \\
\text { Normal }\end{array}$ \\
\hline 4 & Total & 4 & Corrosis biliar & $(-)$ & $\begin{array}{l}\text { Dilatación } \\
\text { parcial conductos } \\
\text { intrabepaticos }\end{array}$ & $\begin{array}{l}\text { Vesícula: Normal } \\
\text { Hepáticos: Atrésicos } \\
\text { Colédoco: Hipoplásico } \\
\text { udenopatías }\end{array}$ \\
\hline 5 & Total & $1 / / 2$ & Hepatitus crónica & $\{-\}$ & $(-)$ & $\begin{array}{l}\text { Vesicula: Atrófica } \\
\text { Heṕ́ticos: }(-) \\
\text { Colédoco: Fibroso }\end{array}$ \\
\hline 6 & $\begin{array}{l}\text { Parcial } 24 \mathrm{~d} \text {. } \\
\text { Total } 6 \mathrm{~d} \text {. }\end{array}$ & 1 & Colestania & $(-)$ & $(-)$ & $\begin{array}{l}\text { Vesicula: Normal } \\
\text { Colédoco: Normal } \\
\text { Hepáticos: Hipoplásicos }\end{array}$ \\
\hline 7 & Parcial & $\begin{array}{c}20 \\
\text { Djas }\end{array}$ & Hepatitis leve & . $\quad+$ & NO & $\mathrm{NO}$ \\
\hline 8 & NO & & NO & + & NO & NO \\
\hline
\end{tabular}


síndrome de Down, sólo se encontró una impregnación difusa del parénquima por pigmentos biliares y hepatocitos vacuolados en las zonas centro lobulillares.

En los 6 lactantes que tenían clínicamente una obstrucción total de vía biliar caracterizada por coluria y acolia persistente (Casos 1 al 6) el examen cintigrafico no mostró excreción de radioisótopo, certificando la obstrucción de esta vía.

La colangiografía transparieto hepática mostró sólo en uno de estos lactantes algunos conductos intrahepáticos con cierto grado de dilatación que eventualmente pudieran constituir puntos de drenaje (Caso 4).

La exploración quirúrgica realizada en los 6 pacientes que presentaban clínicamente obstrucción biliar reveló atrofia completa de la vía extrahepática en tres casos; en éstos las vías estaban transformadas en cordones fibrosos, sin lumen, la vesicula era atrófica sin bilis y había adenopatías para-hiliares (Casos 1, 2 y 5 ).

Ел los Casos 3 y 6 la vía extrahepática era normal, con buen paso de medio de contraste de vesícula a duodeno; uno de éstos ( $\mathrm{N}^{\mathrm{O}}{ }^{6}$ ) fue considerado con ambos conductos hepáticos hipoplásicos, pero con lumen. El paciente restante (Caso 4) presentaba alteración de la via extrahepática de grado intermedio; en efecto, la vesicula era normal, el colédoco hipoplásico, pero con lumen, permitiendo el flujo de vesícula a duodeno, pero ambos conductos hepáticos eran atrésicos, sin lumen. Tambiên existía adenopatía para-hiliar.

En ninguno de tos 6 pacientes fue posible realizar una intervención quirírgica que permitiera un drenaje adecuado del flujo biliar ya que, incluso en el Caso 4 que señalaba cierto grado de dilatación canalicular intrahepática, la exploración del hilio hepático no mostró elementos que justificaran una anastómosis con posibilidades de éxito.

\section{COMENTARIO}

Existen varios aspectos importantes de analizar. Desde luego la alta incidencia de lactantes que en mayor o en menot grado y con lesiones de diversas caracteristicas, aparecen con la vía biliar irremediablemente alterada. Esto adquiere mayor trascendencia cuando la casuistica ha sido reunida en un lapso relativamente breve.

El anallisis de los antecedentes y de los hallazgos de algunos exámenes nos permite comprobar la presencia de varios factores que han sido reconocidos como agentes desencadenantes del síndrome en estudio. Desde luego, uno de ellos (Caso 4) fue un recièn nacido de pretérmino, hijo de madre luética y en quien se comprobó precozmente infección urinaria y probablemente sepsis; todos éstos, factores condicionantes de daño hepático y colestasia. En los otros 6 lactantes y como ha sido habitual en nuestra experiencia, el estudio para Toxoplasmosis, Chagas, Inclusión Citomegálica, Rubeola, y Hepatitis B fue negativo. Por dificultades técnicas no fue posible descartar algún déficit Alfa 1 Antitripsina en esta serie de pacientes. En cambio el estudio histológico del hígado mostró alteraciones características de hepatitis en evolución en 3 lactantes (Casos 1, 5 y 7 ), en dos de eilos intensas y con aspecto de Hepatitis Crónica.

En los 3 casos restantes no existen elementos que permitan identificar algún factor etiológico, salvo la Trisomia 21 que por motivos que no se conocen, se asocia a colestasia crónica en proporción de 14: 1 en relación al lactante normal, y que se encontraba presente en dos pacientes ${ }^{3-9}$.

En otros dos lactantes el cuadro clínico global aparenta tener las características de "Atresia biliar intrahepática" tanto por la normalidad de la vía externa como por la falta de visualización de canalículos intrahepáticos en la colangiografia; sin embargo, esta situación no puede ser asegurada debido a que el estudio histológico fue realizado en un trozo hepático pequeño.

En la técnica de radioisótopo empleada se utilizó el tratamiento previo con fenobarbital, como inductor enzimático ${ }^{8}$, con el fin de poder corregir la colestasia funcional de origen hepatocelular; sin embargo, en ninguno de los 6 lactantes se obtuvo efecto positivo, e incluso, en uno de ellos (Caso 6), la obstrucción total apareció a los 10 dias de tratamiento con fenobarbital.

\section{RESUMEN}

Los resultados obtenidos en el material presentado muestran la concurrencia de varios factores que tienden a confirmar el concepto actual sobre esta patología en el sentido que la ictericia de tipo obstructivo en el lactante menor constituye un síndrome condicionado por una colangiopatía obstructiva, de evolución progresiva que compromete en diferente grado y extensión tanto la vía biliar intra como extrahepática. Esta lesión correspondería a una modalidad reactiva propia del lactante menor frente a diferentes tipos de agresiones al sistema excretor hepático y cuya etiología es posible precisar sólo en un número limitado de pacientes. En este contexto, la técnica con radioisótopos utilizada en el grupo estudiado, se muestra como un procedimiento útil en la pesquisa de permeabilidad del árbol biliar, pero sin un rendimiento superior a la clínica. 


\section{REFERENCIAS}

${ }^{1}$ Hays, D.M. Atresia biliar: Estado actual de confusión. Clín. Quir. N.A. 53: $1257,1973$.

${ }^{2}$ Larrain, $F_{\text {. }}$ Damis, $O$. Ictericia obstructiva del primer trimestre. Estudio evolutivo en 29 lactantes. Rev. Chil. Ped. 45: 427, 1974.

${ }^{3}$ Henriksen, N.T.; Dvablos, P.; Hagenaes, $O$. Cholestatic Jaundice in Infancy. The importance of familial and genetic factors in aetiology and prognosis. Arch. Dis. Child. 56: 622, 1981 .

4 Ghishan, F.X.: Lo Brecque. D.R.; Mitros, F.A.; Younoszai, M.K. The evolving nature of "infantile obstructive cholangiopathy". J. Pediat. 97: 27, 1980.

5 Greene, H.L.; Helinek, G.L.; Moran, R.; OWeill, J. A diagnosis approach to prolonged obstructive Jaundice by 24 hour collection of duodenal fluid. J. Pediat. 95: $412,1979$.
${ }^{6}$ Hirrig, J.; Rickham, P.P. Early differential diagnosis between Neonatal Hepatitis and Biliary Atresia. J. Pediat. Surg. 15: 13, 1980.

Thashimoto, T.; Yura, J. Percutaneous Transhepatic Cholangiography in biliary Atresia with special reference to structure of the intrahepatic bile ducts. J. Pedizt. Surg. 16: 22, 1981.

Mayd, M.: Reba, R; Altmen, R.P. Hepatobiliary Scintigraphy with $99^{\mathrm{m}}$ Tc - PIPIDA in the evaluation of Neonatal Jaundice. Pediat. 67: 140, 1981.

$\rightarrow$ Alpert, L.I.; Strauss, L.; Hoschhorn, $X$. Neonatal Hepatitis and Biliary Atresia associated with autosomal Trysomy Syndrome. Gastroenterology 54: 14B, 1968.

$10 \mathrm{Ed}$. Progresive extrahepatic Biliary obstruction of the newborn. J. Pediat. Surg. 10: 169, 1975.

11 Lunding, B.H. Considerations of the pathogenesis of Neonatal Hepatitis, biliary atresia and choledochal cyst. The concept of infantile obstructive cholangiopathy. Progr. Ped. Surg. 6: 113, 1974. 